\title{
Revista Colombiana de

\section{Desenlaces a un año en pacientes con síncope de alto y bajo riesgo}

\author{
Alejandro Olaya Sánchez ${ }^{\mathrm{a}}$, Miguel Tejeda Camargo ${ }^{\mathrm{b}, \mathrm{c}, *}$, Luis Cárcamo ${ }^{\mathrm{a}}$, \\ Diana Vargas ${ }^{a}$, Andrés Díaz ${ }^{a}$ y Guillermo Mora ${ }^{\mathrm{d}, \mathrm{e}}$
}

\author{
a Departamento de Cardiología, Fundación Universitaria de Ciencias de la Salud - Hospital de San José de Bogotá, Bogotá, \\ Colombia \\ b Electrofisiología Cardiovascular, Universidad de la Sabana, Bogotá, Colombia \\ "Centro Internacional de Arritmias "Andrea Natale" - Fundación Cardioinfantil - Instituto de Cardiología, Bogotá, Colombia \\ 'Universidad Nacional de Colombia, Bogotá, Colombia \\ e Servicio de Electrofisiología, Fundación Santa Fe de Bogotá, Bogotá, Colombia
}

Recibido el 13 de mayo de 2019; aceptado el 20 de septiembre de 2019

Disponible en Internet el 1 de mayo de 2020

\author{
PALABRAS CLAVE \\ Síncope; \\ Riesgo; \\ Mortalidad; \\ Recurrencia; \\ Hospitalización
}

\begin{abstract}
Resumen
Introducción: el síncope es un síntoma complejo de evaluar, que además representa un reto diagnóstico; la estratificación inadecuada del riesgo de los pacientes puede conllevar mal uso de los recursos en salud y aumentar así el costo derivado de la atención.

Objetivo: comparar la incidencia de desenlaces a un año en pacientes con síncope de alto y bajo riesgo en cuatro hospitales de Bogotá.

Metodología: estudio de cohorte retrospectiva, en el que se incluyeron pacientes mayores de 18 años que consultaron a urgencias por síncope, y que fueron seguidos desde febrero de 2013 hasta julio de 2015. Fueron clasificados en bajo y alto riesgo según la escala de Martin et al. Se hizo seguimiento telefónico para evaluar los desenlaces a un año.

Resultados: los pacientes de alto riesgo tienen mayor frecuencia de mortalidad, recurrencia del síncope, requerimiento de hospitalización, eventos neurológicos y necesidad de intervención cardiovascular. La mortalidad global está alrededor del $6 \%$ y en el subgrupo de pacientes de mayor puntaje (4 puntos) asciende al $16,6 \%$. La mayoría de eventos se presentaron en los primeros 6 meses de seguimiento. La mortalidad y la necesidad de intervención cardiovascular tuvieron un incremento de su frecuencia a medida que aumentó el puntaje de la escala.

Conclusión: los pacientes clasificados en alto riesgo según la escala de Martin et al. tienen mayor frecuencia de desenlaces adversos a un año de seguimiento, y por tanto podrían beneficiarse de un estudio más amplio y expedito de la causa del síncope.

(c) 2019 Sociedad Colombiana de Cardiología y Cirugía Cardiovascular. Publicado por Elsevier España, S.L.U. Este es un artículo Open Access bajo la licencia CC BY-NC-ND (http:// creativecommons.org/licenses/by-nc-nd/4.0/).
\end{abstract}

\footnotetext{
* Autor para correspondencia.

Correos electrónicos: migueljosetejeda@hotmail.com (M. Tejeda Camargo), gmorap@unal.edu.co (G. Mora).
} 


\section{KEYWORDS}

Syncope;

Risk;

Mortality;

Recurrence;

Hospitalisation

\section{Introducción}

El síncope se define como una pérdida transitoria del conocimiento debido a una hipoperfusión cerebral global transitoria caracterizada por inicio rápido, duración corta y recuperación espontánea completa ${ }^{1}$. Es un síntoma frecuente, con una prevalencia del $42 \%$, y una incidencia anual del $6 \%^{2}$; se presenta como síntoma principal en el 1 al $5 \%$ de los pacientes que consulta al servicio de urgencias $^{3}$ y tiene una presentación bimodal alcanzando la mayor frecuencia entre los 10 y 30 años de edad y luego de los 65 años $^{1,4}$. Su recurrencia está alrededor del $13,5 \%{ }^{1}$ en cuyo caso la estenosis aórtica, la disfunción renal, la edad avanzada y los medicamentos hipotensores son unos de los factores de riesgo más asociados ${ }^{5}$. La causa más frecuente de síncope es el reflejo, también conocido como síncope vasovagal, seguido de la causa cardiogénica y de la hipotensión ortostática6 ${ }^{6}$.

Este síntoma es responsable del 1 al $5 \%^{7}$ de todas las causas de consulta al servicio de urgencias con una frecuencia de hospitalización cercana al $50 \%^{2}$, siendo estas innecesarias hasta en la mitad de los $\operatorname{casos}^{8}$. En Estados Unidos un estudio estimó que la hospitalización derivada de este diagnóstico tiene un costo anual de 2.4 millones de dólares (IC 95\% 2,2 a 2,6), un costo medio de 5.400 dólares por hospitalización y es tan alta como 8.500 dólares según otros estudios ${ }^{9,10}$, costos comparables con enfermedades como asma, enfermedad pulmonar obstructiva crónica e infección por virus de la inmunodeficiencia humana, cifras que demuestran el alto consumo de recursos y la necesidad de optimizar el abordaje diagnóstico y terapéutico ${ }^{9}$.
Dado lo anterior, se requiere la implementación de escalas pronóstico que permitan predecir desenlaces como mortalidad, recurrencia, daño físico, necesidad de intervenciones, entre otros, a corto y largo plazo, lográndose así dar prioridad a la hospitalización y a la administración de los recursos en salud ${ }^{11}$. Existen varias escalas para la predicción del pronóstico en pacientes con síncope; sin embargo, hay subutilización de las mismas tal vez por desconocimiento de éstas, por la falta de certeza de la utilidad o por ausencia de un consenso acerca de la forma de su uso ${ }^{12}$.

Este estudio comparó la incidencia de desenlaces a un año de seguimiento luego del primer episodio de síncope en pacientes clasificados como bajo y alto riesgo según la escala de Martin et al. ${ }^{13}$ en cuatro hospitales de Bogotá, Colombia. Las variables de esta escala incluyen un electrocardiograma anormal en el departamento de urgencias, historia de arritmia ventricular, antecedentes de insuficiencia cardíaca congestiva y edad mayor a 45 años. Entre los desenlaces a valorar en este estudio están la mortalidad, la recurrencia del síncope, la necesidad de intervenciones cardiovasculares y la presencia de eventos neurológicos.

\section{Materiales y métodos}

Se llevó a cabo un estudio de cohorte retrospectivo, cuyos pacientes fueron identificados mediante una base de datos de una cohorte recolectada previamente en los servicios de urgencias de cuatro hospitales de Bogotá, Colombia, desde febrero de 2013 hasta julio de 2015. Se hizo un muestreo consecutivo, por lo que no se requirió cálculo de tamaño de la muestra y se ingresaron todos los pacientes de la base de 
datos descrita que cumplieron los criterios de elegibilidad y que aceptaron participar en el estudio.

Se incluyó a todos aquellos que consultaron a urgencias por síncope, con edad mayor de 18 años y que aceptaron participar en la investigación. Se excluyeron aquellos con historia de déficit cognitivo o del lenguaje y trauma previo a la pérdida de conciencia.

Los pacientes se estratificaron mediante la escala de Martin et al. definiendo como bajo riesgo aquellos con ausencia de factores y como alto riesgo quienes tuvieran uno o más. Fueron contactados vía telefónica con el fin de solicitar la aceptación para participar en el estudio y evaluar los desenlaces a 365 días.

Los desenlaces tenidos en cuenta fueron mortalidad, recurrencia del síncope, requerimiento de intervención cardiovascular, eventos neurológicos y necesidad de nueva hospitalización. Durante el análisis se describieron las características demográficas y clínicas de los participantes del estudio y las variables de la escala de Martin et al. La incidencia de los desenlaces se calculó considerando en el numerador los casos nuevos y en el denominador la población en riesgo de presentar el desenlace, en este caso, la totalidad de pacientes incluidos. Para comparar las tasas de incidencia de desenlaces entre los pacientes de alto y bajo riesgo de mortalidad, según la escala de Martin et al. y teniendo en cuenta que los eventos pueden presentarse en varias ocasiones durante el seguimiento, se llevó a cabo un análisis de supervivencia para eventos múltiples.

\section{Ética}

Esta investigación está dentro de los paráetros internacionales para estudios con seres humanos, sigue las normas internacionales del código de Helsinki, código de Núremberg y la reglamentación colombiana sustentada en la resolución 8430 del año 1993, considerada como investigación sin riesgo. El protocolo fue avalado por el comité de investigaciones y comité de ética en investigación con seres humanos de la Fundación Universitaria de Ciencias de la Salud. Así mismo, fue aprobado por el comité de ética de los hospitales participantes.

\section{Estadística}

\section{Descripción de la muestra de participantes}

Las variables de tipo cualitativo se describen por medio de frecuencias absolutas y relativas, en tanto que las variables cuantitativas por medio de medias y desviaciones estándar o medianas y rangos intercuartílicos de acuerdo con su distribución.

\section{Descripción de las variables de la escala de Martin et al. en la muestra de pacientes}

Por medio de frecuencias absolutas y relativas se determinó el número de pacientes que cumplieron con criterios positivos de cada variable de la escala de Martin et al. (electrocardiograma anormal en el departamento de urgencias, historia de arritmia ventricular, antecedentes de

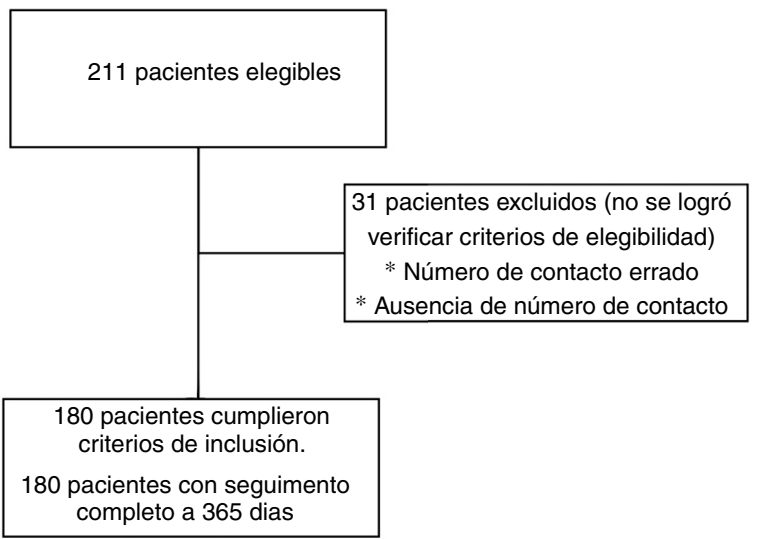

Figura 1 Pacientes elegibles y con seguimiento completo.

insuficiencia cardíaca congestiva y edad mayor de 45 años). Posteriormente, se determinó la frecuencia absoluta y relativa de los pacientes jerarquizados de acuerdo con el algoritmo de clasificación de la escala (0-1-2-3-4), definiendo como bajo riesgo los calificados con $0 \mathrm{y}$ alto riesgo los que tuvieron una puntuación de 1 o más. Se presentó la frecuencia (absoluta y relativa) de pacientes catalogados de acuerdo con el algoritmo de clasificación de la escala según el sexo.

\section{Incidencia de eventos adversos en la muestra de pacientes}

Se calculó la incidencia de eventos adversos (IEA) a 365 días considerando en el numerador los casos nuevos y en el denominador la población en riesgo de presentar el desenlace, en este caso, la totalidad de pacientes incluidos.

\section{Comparación de la incidencia de eventos adversos entre grupos de pacientes definidos por la escala de Martin et al.}

Para comparar las tasas de incidencia de eventos adversos entre los pacientes en alto y bajo riesgo de mortalidad según la escala de Martin et al. y teniendo en cuenta que los eventos pueden presentarse en varias ocasiones durante el seguimiento, se llevó a cabo un análisis de supervivencia para eventos múltiples. Para esto se usó un modelo paramétrico, se hicieron curvas de supervivencia y se calcularon medidas de asociación.

\section{Resultados}

Se identificaron 211 pacientes que consultaron a urgencias por síncope desde febrero de 2013 hasta julio de 2015. De este total de pacientes se logró la verificación de criterios de inclusión y exclusión en 180, y todos aceptaron participar en el estudio. Se hizo seguimiento telefónico a los 180 participantes (fig. 1).

La edad promedio de la población fue 63,01 años (desviación estándar de 20,79 años); la edad mínima fue 17 y la máxima 93 años. En la tabla 1 se describen las características básicas de la población. 
Tabla 1 Características basales de la población participante

\begin{tabular}{|c|c|}
\hline Variable & $\begin{array}{l}\text { Frecuencia } \\
n=180\end{array}$ \\
\hline Edad & $\begin{array}{l}\text { Mediana } 69 \\
\text { años (RIC } 50 \text { a } \\
80 \text { años) }\end{array}$ \\
\hline \multicolumn{2}{|l|}{ Género } \\
\hline Femenino & $99(55 \%)$ \\
\hline Masculino & $81(45 \%)$ \\
\hline \multicolumn{2}{|l|}{ Raza * } \\
\hline Negra & $3(1,69 \%)$ \\
\hline Mestiza & $92(51,69 \%)$ \\
\hline Blanca & $81(45,51 \%)$ \\
\hline Otra & $2(1,12 \%)$ \\
\hline \multicolumn{2}{|c|}{ Electrocardiograma anormal } \\
\hline No & $88(48,89 \%)$ \\
\hline $\mathrm{Si}$ & $92(51,11 \%)$ \\
\hline \multicolumn{2}{|c|}{ Historia clínica de arritmia ventricular } \\
\hline No & $159(88,33 \%)$ \\
\hline $\mathrm{Si}$ & $21(11,67 \%)$ \\
\hline \multicolumn{2}{|c|}{ Historia clínica de insuficiencia cardiaca } \\
\hline No & $148(82,22 \%)$ \\
\hline $\mathrm{Si}$ & $32(17,78 \%)$ \\
\hline \multicolumn{2}{|c|}{ Historia clínica de enfermedad cardiovascular } \\
\hline No & $111(61,67 \%)$ \\
\hline $\mathrm{Si}$ & $69(38,33 \%)$ \\
\hline \multicolumn{2}{|l|}{ Síncope } \\
\hline Sin pródromo & $77(42,78 \%)$ \\
\hline Con pródromo & $103(57,22 \%)$ \\
\hline \multicolumn{2}{|c|}{ Puntaje Escala Martin } \\
\hline 0 & $28(15,56 \%)$ \\
\hline 1 & $56(31,11 \%)$ \\
\hline 2 & $65(36,11 \%)$ \\
\hline 3 & $25(13,89 \%)$ \\
\hline 4 & $6(3,33 \%)$ \\
\hline
\end{tabular}

La mayoría de pacientes del estudio correspondía al sexo femenino (55\%) y a la raza mestiza $(51,69 \%)$; casi la mitad de estos tuvo electrocardiograma anormal $(51,11 \%)$, definido por la presencia de disturbios del ritmo (taquiarritmias), desorden de la conducción (bloqueo aurículo-ventricular de segundo y tercer grado, bloqueos de rama del has de His, desviación del eje a la izquierda), hipertrofia de cavidades o hallazgos compatibles con infarto agudo de miocardio previo. El antecedente conocido de arritmias ventriculares, falla cardiaca y enfermedad cardiovascular no superó individualmente el 40\%, y más de la mitad de los pacientes refirió pródromos antes del episodio sincopal.

Los desenlaces más frecuentes fueron síncope recurrente $(21,6 \%)$ y necesidad de intervención cardiovascular (26,67\%); la mortalidad fue cercana al $6 \%$ y los eventos neurológicos ocuparon el lugar inferior en frecuencia con un $4 \%$ (tabla 2). La mayoría de pacientes fueron clasificados como alto riesgo con un puntaje de 2 (tabla 1 ).
Tabla 2 Frecuencia de desenlaces

\begin{tabular}{lll}
\hline Variable & Frecuencia (n 180) & Porcentaje \\
\hline Muerte & 11 & $6,11 \%$ \\
Recurrencia & 39 & $21,6 \%$ \\
Rehospitalización & 20 & $11,11 \%$ \\
Necesidad de intervención & 48 & $26,67 \%$ \\
Eventos neurológicos & 7 & $3,89 \%$ \\
\hline
\end{tabular}

La mortalidad fue mayor en aquellos clasificados como alto riesgo por la escala de Martin et al. y alcanzó el 16,6\% en aquellos con un puntaje de 4 (fig. 2).

Aunque la recurrencia del síncope fue más frecuente en los pacientes de alto riesgo no se evidenció una relación constante con el incremento del puntaje en la escala (fig. 3). Proporcionalmente, la necesidad de una nueva hospitalización después del primer episodio de síncope fue mayor en los pacientes de bajo riesgo (fig. 4).

La necesidad de intervención cardiovascular tuvo un comportamiento similar al de la mortalidad, con un incremento de la frecuencia del evento a medida que el puntaje de la escala aumentó; sin embargo, los pacientes con mayor puntaje (4 puntos) tuvieron un descenso en la frecuencia del desenlace, aunque sosteniéndose por encima de los pacientes de bajo riesgo (figs. 5 a 8).

La mediana de supervivencia para cualquier evento fue de 45 días (RIC 9-100), muerte 78 días (RIC 29-158), recurrencia 81 días (RIC 32-104), necesidad de una nueva hospitalización 71 días (RIC 11-92), necesidad de intervención cardiovascular 7 días (RIC 7-41) y eventos neurológicos 132 días (RIC 90-164) (figs. 9 a 14).

El riesgo $=0$ es bajo y el riesgo $=1$ es alto (puntajes 1,2 , 3, 4 en la escala de Martin et al.).

$\psi$ Solo se grafica la curva de los pacientes de alto riesgo, pues ninguno en el grupo de bajo riesgo murió en el término del seguimiento. Riesgo $=0$ es bajo y el riesgo $=1$ es alto (puntajes 1, 2, 3, 4 en la escala Martin et al.)

El riesgo $=0$ es bajo y el riesgo $=1$ es alto (puntajes 1,2 , 3, 4 en la escala de Martin et al.).

$\mathrm{El}$ riesgo $=0$ es bajo y el riesgo $=1$ es alto (puntajes 1,2 , 3, 4 en la escala de Martin et al.).

$¥$ Aquí solo aparece graficada una línea porque no hubo pacientes de bajo riesgo que tuvieran el desenlace evento neurológico. Los 7 pacientes que presentaron evento neurológico fueron considerados en alto riesgo según la escala de Martín et al. (puntaje 1 o más). El riesgo $=0$ es bajo y el riesgo $=1$ es alto (puntajes 1, 2, 3, 4 en la escala de Martin et al.).

\section{Discusión}

El síncope es un síntoma difícil de evaluar, que además constituye un reto diagnóstico en el servicio de urgencias. La estratificación inadecuada del riesgo puede llevar al mal uso de los recursos en salud e incrementar así el costo derivado de la atención ${ }^{9}$. En este estudio se comparó la incidencia de desenlaces entre pacientes con síncope de bajo y alto riesgo clasificados según la escala de Martin et al.

Los hallazgos sugieren que en los pacientes clasificados como alto riesgo, según la escala en mención ${ }^{13}$, hay mayor 


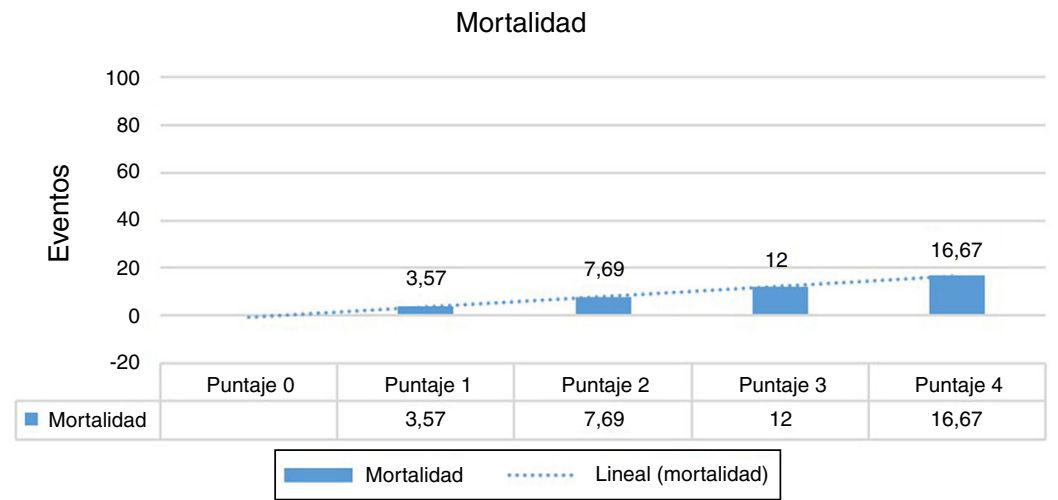

Figura 2 Frecuencia de mortalidad dividida en grupos de pacientes según el puntaje de la escala de Martin et al.

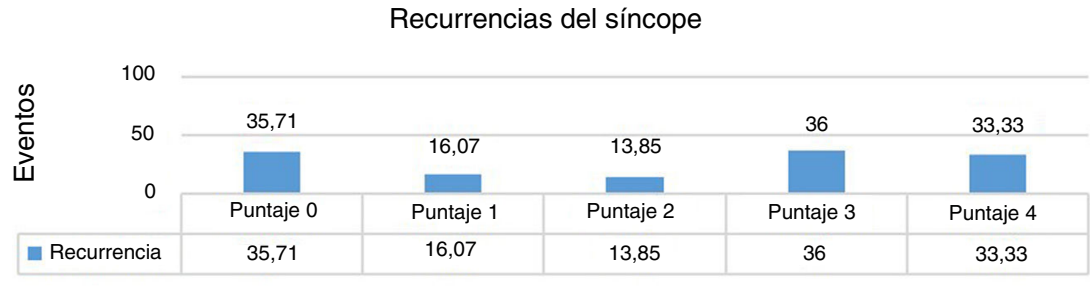

Recurrencia

Figura 3 Recurrencia del síncope dividida en grupos de pacientes según el puntaje de la escala de Martin et al.

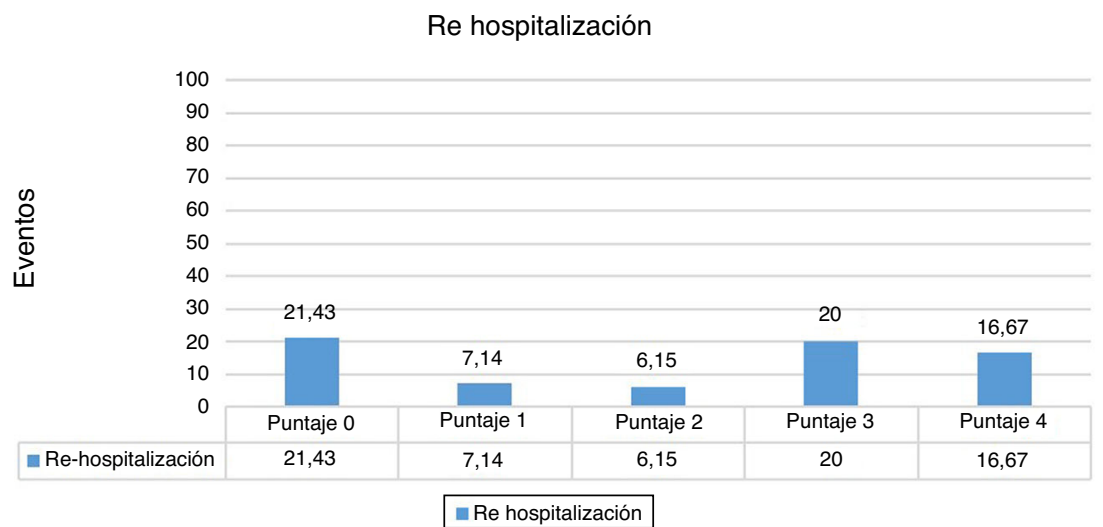

Figura 4 Necesidad de rehospitalización dividida en grupos de pacientes según el puntaje de la escala de Martin et al.

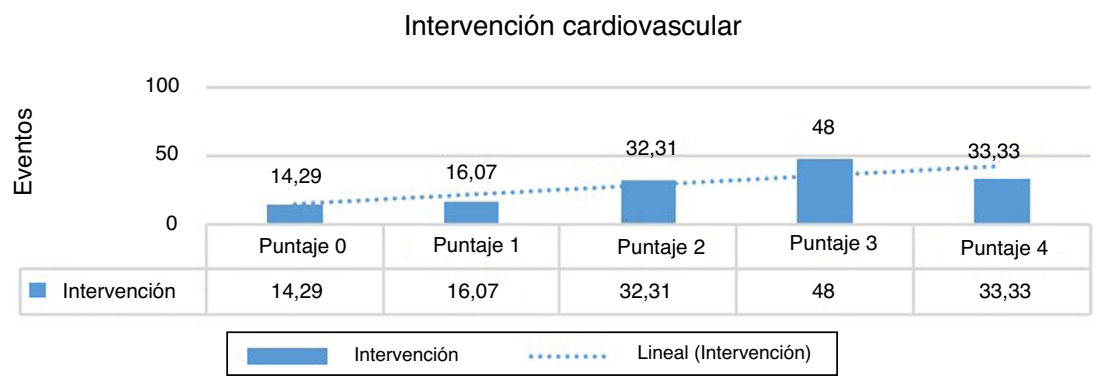

Figura 5 Necesidad de intervención cardiovascular dividida en grupos de pacientes según el puntaje de la escala de Martin et al. 


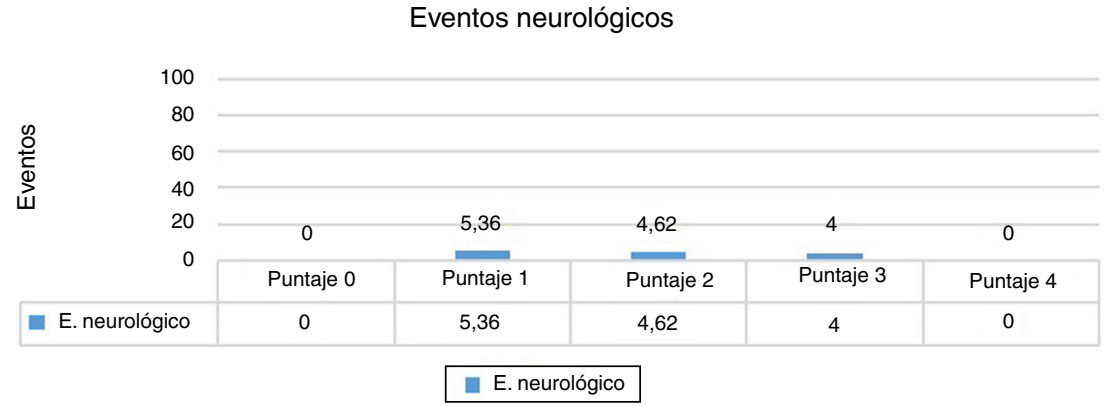

Figura 6 Eventos neurológicos divididos en grupos de pacientes según el puntaje de la escala de Martin et al.

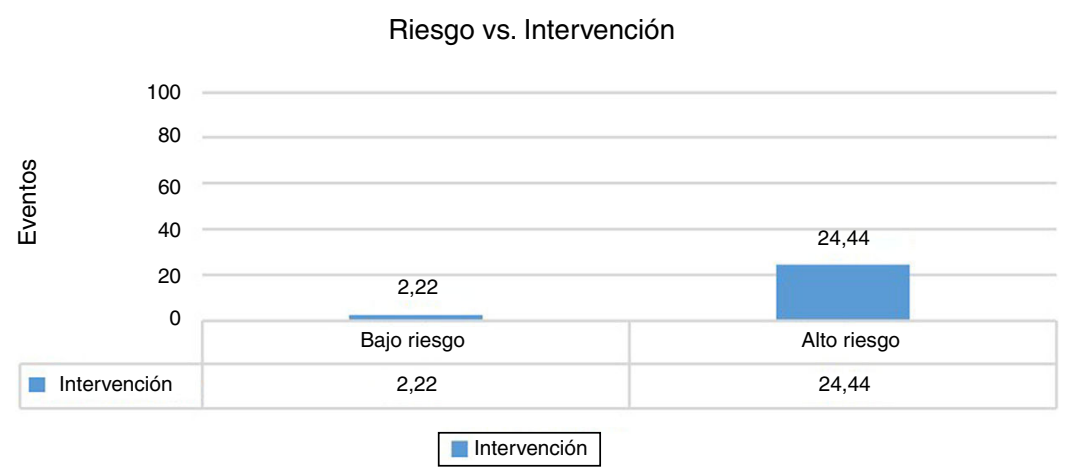

Figura 7 Comparación del desenlace requerimiento de intervención cardiovascular en pacientes de bajo y alto riesgo según la escala de Martin et al.

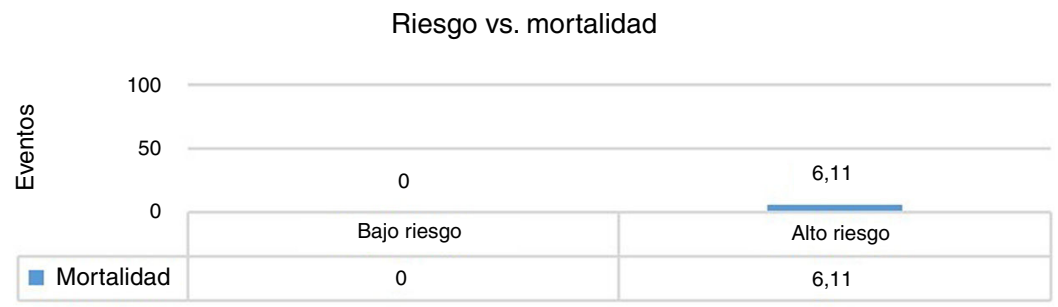

Mortalidad

Figura 8 Comparación del desenlace mortalidad en pacientes de bajo y alto riesgo según la escala de Martin et al.

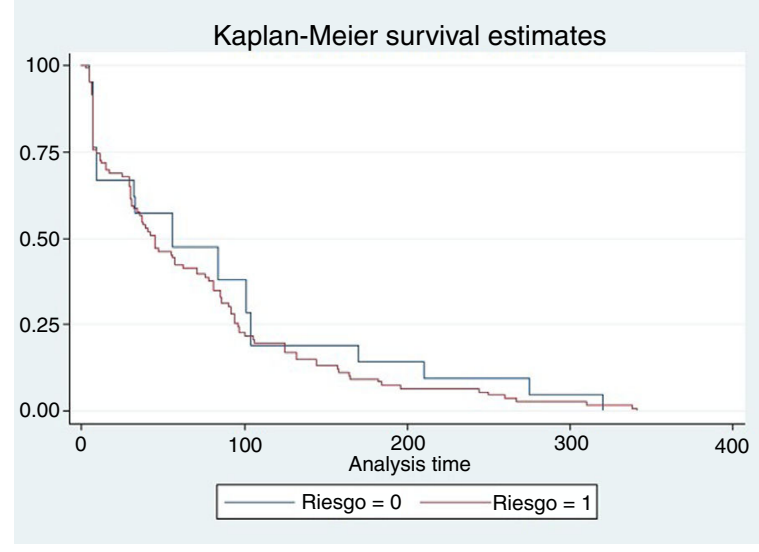

Figura 9 Curva de supervivencia para cualquier evento según el riesgo.

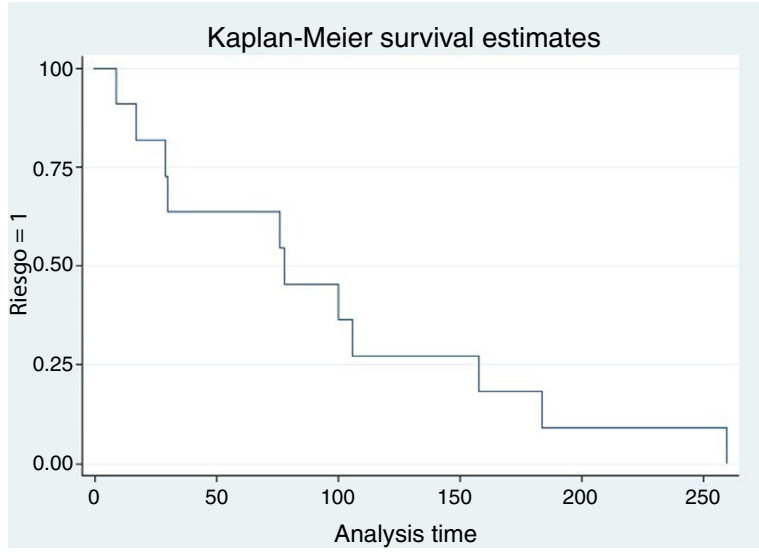

Figura 10 Curva de supervivencia para evento: muerte según riesgo $\psi$. 


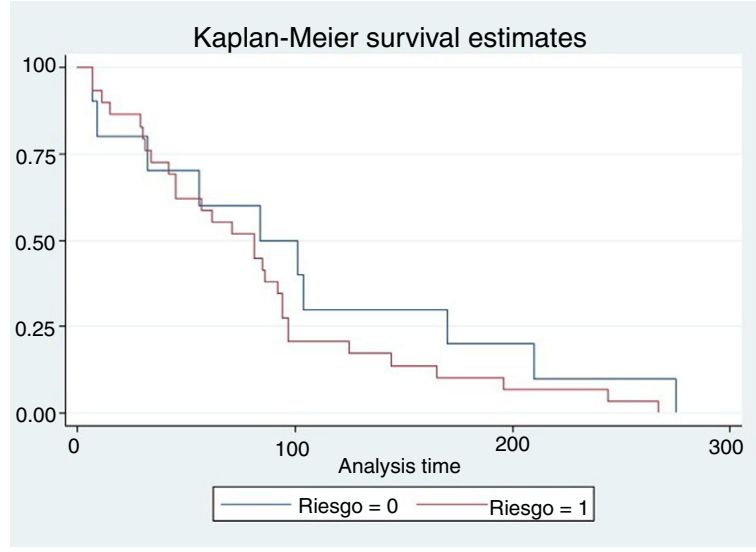

Figura 11 Curva de supervivencia para evento: Recurrencia.

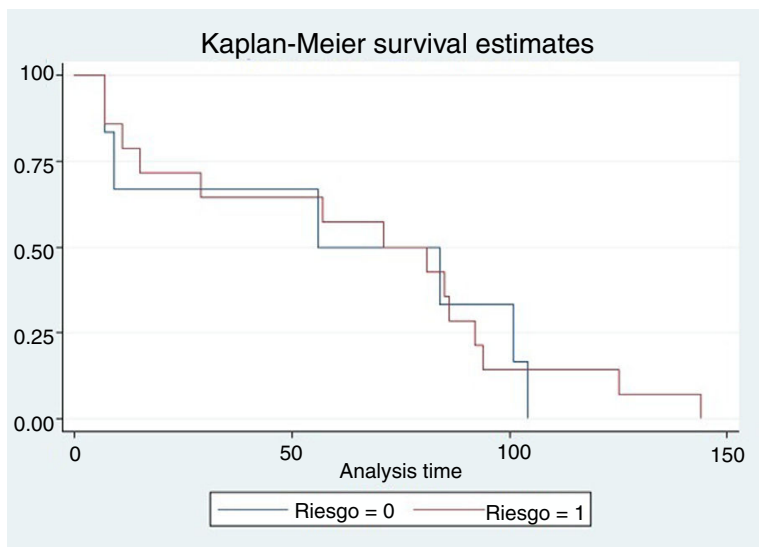

Figura 12 Curva de supervivencia para evento: rehospitalización según el riesgo.

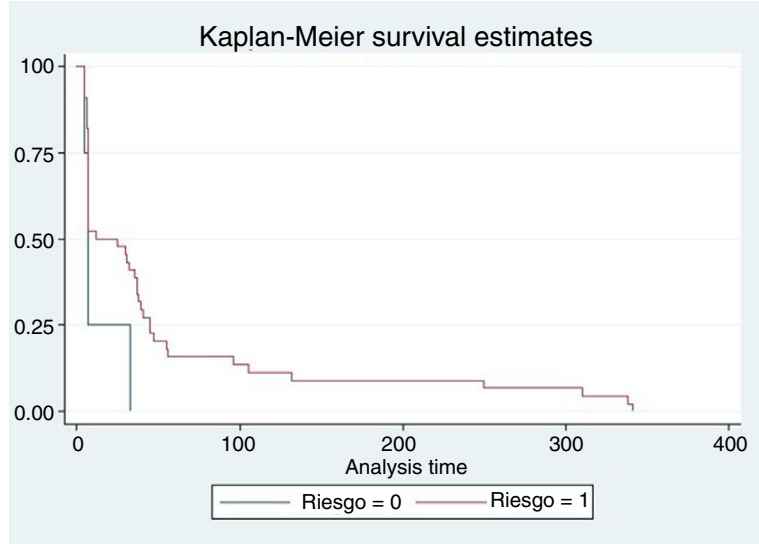

Figura 13 Curva de supervivencia para evento: intervención cardiovascular según el riesgo.

frecuencia de los desenlaces descritos; en este sentido, la mortalidad y la necesidad de intervención cardiovascular son los que se incrementan en frecuencia a medida que se aumenta el puntaje de la escala. De 211 pacientes que se identificaron en la base de datos inicial solo 180 cumplieron los criterios de inclusión y en todos fue posible realizar el seguimiento a un año.

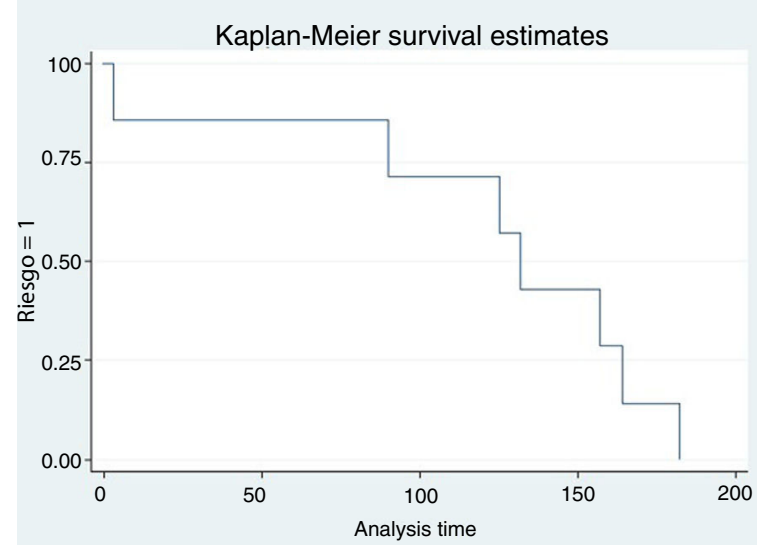

Figura 14 Curva de supervivencia para evento: Evento neurológico según el riesgo $¥$. El riesgo $=0$ es bajo y el riesgo $=1$ es alto (puntajes 1, 2, 3, 4 en la escala de Martin et al.).

La mayor frecuencia en el género femenino y la edad promedio evidenciada concuerdan con estudios previos acerca de la epidemiología del síncope ${ }^{14}$ y con el estudio original de la escala valorada ${ }^{13}$. El rango de edad en este estudio es amplio, lo cual permite que los resultados puedan considerarse en varios grupos de pacientes.

Aproximadamente la mitad de los pacientes tuvo un hallazgo electrocardiográfico anormal, casi en similar proporción que lo ocurrido en la cohorte de validación de la escala de Martin $^{13}$. Tal vez este hallazgo esté relacionado con el hecho de que los pacientes que acuden al servicio de urgencias posiblemente tienen más comorbilidades cardiovasculares, además de mayor sensibilidad a la hora de la lectura del electrocardiograma por parte del personal especializado encargado de la recolección de los datos de la cohorte inicial. A la luz de la evidencia clínica actual debe plantearse una consideración adicional y es que los hallazgos anormales electrocardiográficos condicionan por sí mismos un factor de riesgo mayor hacia desenlaces cardiovasculares desfavorables ${ }^{1}$.

Cuando se hizo la estratificación por grupos según el puntaje, la mayoría de los pacientes fueron clasificados como alto riesgo (puntaje mayor o igual a 1) alcanzando un $84 \%$ y solo como bajo riesgo el $16 \%$ restante; esto deja claro que la clasificación utilizada en el estudio puede ser útil en la valoración de los pacientes que consultan a los servicios de urgencias y sugiere que los de alto riesgo son quienes cuentan con más probabilidad de tener condiciones potencialmente mortales. De la estratificación el mayor porcentaje de población se encuentra con un puntaje de 2 , representado por un $36,1 \%$ de la población estudiada. Como limitación importante se evidencia que los pacientes clasificados con el mayor puntaje no tienen una muestra robusta; solo 3 pacientes en la cohorte cumplen con estas características, lo que podría reducir la posibilidad de conclusiones contundentes en este grupo.

La mortalidad global en la cohorte alcanza un 6,1\%. Cuando se hace una estratificación de los pacientes por la sumatoria de los factores de riesgo definidos por la escala se evidencia un incremento de esta a medida que aumenta el puntaje (fig. 2) y al evaluar los datos según el puntaje aquellos con las cuatro variables tienen una mortalidad 
que asciende al 16,6\%; es por esto que se considera que esta escala podría ser aplicada en la población colombiana para definir quiénes pueden beneficiarse de una valoración oportuna con el objetivo de minimizar este resultado. Adicionalmente, la necesidad de intervención cardiovascular tuvo un comportamiento similar. Al comparar el desenlace mortalidad con lo reportado por Martin et al. en la cohorte de validación de la escala se encontró menor frecuencia del desenlace, pues reportan una frecuencia de $27,3 \%{ }^{12,13}$ para los pacientes con tres a cuatro factores de riesgo, hallazgo que podría estar relacionado con el menor tamaño de la muestra del estudio. Lo que sí presenta una similitud es el incremento de la frecuencia del evento a medida que el puntaje es mayor.

La recurrencia global del síncope luego del primer episodio es de $21,6 \%$ y cuando se compara bajo vs. alto riesgo es más frecuente en aquellos clasificados como riesgo elevado; sin embargo, no se observó una relación de la severidad del puntaje con la frecuencia del desenlace, hallazgos que se asemejan con la necesidad de hospitalización y los eventos neurológicos.

La mediana de supervivencia para cualquier evento fue de 45 días con un RIC de 9 a 100 días (fig. 9); de esta forma, se entiende que la mitad de los eventos ocurren de manera temprana, antes del segundo mes de seguimiento, y casi la totalidad de estos (75\%) antes del cuarto mes, lo cual podría sugerir que la mayor utilidad de la escala es la detección de desenlaces tempranos (menos de 6 meses).

Al analizar los eventos de forma individual la mortalidad tiene una mediana de 78 días con un RIC de 29 a 158 días (fig. 10), resultado que propone que la mayoría de muertes ocurren en los primeros 6 meses de seguimiento, de modo que los pacientes clasificados como alto riesgo con la escala de Martin et al. deben tener un estrecho seguimiento, en especial en el intervalo de tiempo mencionado, a fin de favorecer la canalización de recursos y herramientas diagnósticas en la búsqueda de la etiología y facilitar así la gestión de los pacientes que ingresan por síncope al servicio de urgencias.

Cabe resaltar que la necesidad de intervención cardiovascular, como el implante de un dispositivo, ocurrió en su mayoría antes del mes y medio de seguimiento (la mitad de los casos se presentaron en los primeros 7 días), considerándose de esta forma que los pacientes libres de este evento luego del segundo mes de seguimiento tienen probablemente menos frecuencia de este a un año (fig. 13).

Los factores de riesgo utilizados en esta escala para la estratificación de los pacientes son similares a los usados en otras escalas, en los que la edad avanzada ${ }^{15}$, el antecedente de enfermedad cardiovascular ${ }^{16}$ y la falla cardiaca ${ }^{13}$ se correlacionaron con desenlaces adversos a largo plazo; incluso varias escalas de predicción solapan estos factores como determinantes en la predicción de riesgo, tanto a corto como a largo plazo ${ }^{17}$.

La limitación más importante del estudio es el sesgo de memoria pues, al ser una base de datos recolectada hace más de un año, es posible que los pacientes omitieran un desenlace o la fecha exacta del mismo. Esta limitación se puede ver mitigada con el hecho de que los desenlaces se consideren de gran impacto y que, por ende, la probabilidad de recordarlos sea mayor. Otra limitación importante está en relación con la restricción de los resultados solo al ámbito de urgencias y no al paciente con síncope que acude a la consulta externa; además, el estudio se llevó a cabo en hospitales de cuarto nivel de complejidad y los pacientes atendidos en centros de atención primaria, posiblemente con menor complejidad, no están representados en la cohorte.

Como se expresó anteriormente, el síncope es un síntoma complejo, con múltiples etiologías a menudo difíciles de identificar al hacer la historia clínica y la valoración paraclínica inicial; hasta en el $60 \%$, 18 de los pacientes no se manifiesta una causa clara luego de la aproximación inicial, razón por la cual es necesario estratificar a aquellos sobre quienes pesa el mayor riesgo de desenlaces ${ }^{14,19}$. En consecuencia, se propone un abordaje sistemático en el servicio de urgencias, que inicie por la confirmación de la definición del síntoma y que cumpla las características operativas de la definición para continuar con el análisis clínico, descartando así otras condiciones que pueden simular esta condición, hacer la valoración inicial de la historia clínica, el examen físico (incluir la prueba de ortostatismo), analizar el electrocardiograma - en este momento, si existe una causa clara, se debe tratar (por ejemplo reducción de medicamentos hipotensores si se documenta ortostatismo) - y en el caso contrario aplicar la escala de riesgo de Martin et al. con el objetivo de identificar a los pacientes de mayor riesgo que se beneficiarán de un estudio clínico más expedito y probablemente intrahospitalario.

Con base en los resultados de este estudio se pueden proponer investigaciones futuras que tengan un diseño epidemiológico con más poder para aseverar los resultados de esta exploración. Se sugiere que la escala de Martin et al. es útil para estratificar pacientes en el servicio de urgencias, y que además sirve de apoyo en la generación de guías de práctica clínica, con miras a optimizar los recursos a través de la resolución expedita de los pacientes que consultan con este síntoma.

\section{Financiación}

Ninguna.

\section{Conflictos de interés}

Ninguno.

\section{Agradecimientos}

A Claudia Irene Ibáñez Antequera y a Luisa María Montoya Quesada por su apoyo, paciencia y asesoría en la ejecución del trabajo.

\section{Bibliografía}

1. Shen WK, Sheldon RS, Benditt DG, Cohen MI, Forman DE, Goldberger ZD, et al. 2017 ACC/AHA/HRS Guideline for the Evaluation and Management of Patients With Syncope: Executive Summary: A Report of the American College of Cardiology/American Heart Association Task Force on Clinical Practice Guidelines, and the Heart Rhythm Society. J Am Coll Cardiol. 2017;70:620-63. 
2. da Silva RM. Syncope: epidemiology, etiology, and prognosis. Front Physiol. 2014;5:471.

3. Runser LA, Gauer RL, Houser A. Syncope: evaluation and differential diagnosis. Am Fam Physician. 2017;95:303-12.

4. Moya A, Sutton R, Ammirati F, Blanc JJ, Brignole M, Dahm JB, et al. Guidelines for the diagnosis and management of syncope (version 2009). Eur Heart J. 2009;30:2631-71.

5. Ruwald MH, Hansen ML, Lamberts M, Hansen CM, Højgaard MV, Køber L, et al. The relation between age, sex, comorbidity, and pharmacotherapy and the risk of syncope: a Danish nationwide study. Europace. 2012;14:1506-14.

6. Kenny RA, Bhangu J, King-Kallimanis BL. Epidemiology of syncope/collapse in younger and older Western patient populations. Prog Cardiovasc Dis. 2013;55:357-63.

7. Smars PA, Decker WW, Shen WK. Syncope evaluation in the emergency department. Curr Opin Cardiol. 2007;22:44-8.

8. McCarthy F, De Bhladraithe S, Rice C, MCMahon CG, Geary U, Plunkett PK, et al. Resource utilisation for syncope presenting to an acute hospital Emergency Department. Ir J Med Sci. 2010;179:551-5.

9. Sun BC, Emond JA, Camargo CA. Direct medical costs of syncope-related hospitalizations in the United States. Am J Cardiol. 2005;95:668-71.

10. Alshekhlee A, Shen WK, Mackall J, Chelimsky TC. Incidence and mortality rates of syncope in the United States. Am J Med. 2009;122:181-8.

11. Shen WK, Traub SJ, Decker WW. Syncope management unit: evolution of the concept and practice implementation. Prog Cardiovasc Dis. 2013;55:382-9.
12. Puppala VK, Akkaya M, Dickinson O, Benditt DG. Risk Stratification of Patients Presenting with Transient Loss of Consciousness. Cardiol Clin. 2015;33:387-96.

13. Martin TP, Hanusa BH, Kapoor WN. Risk stratification of patients with syncope. Ann Emerg Med. 1997;29:459-66.

14. Soteriades ES, Evans JC, Larson MG, Chen MH, Chen L, Benjamin EJ, et al. Incidence and prognosis of syncope. N Engl J Med. 2002;347:878-85.

15. Costantino G, Perego F, Dipaola F, Borella M, Galli A, Cantoni $\mathrm{G}$, et al. Short- and long-term prognosis of syncope, risk factors, and role of hospital admission: results from the STePS (Short-Term Prognosis of Syncope) study. J Am Coll Cardiol. 2008;51:276-83.

16. Serrano LA, Hess EP, Bellolio MF, Murad MH, Montori VM, Erwin $\mathrm{PJ}$, et al. Accuracy and quality of clinical decision rules for syncope in the emergency department: a systematic review and meta-analysis. Ann Emerg Med. 2010;56:362-73, e1.

17. Sheldon RS, Morillo CA, Krahn AD, O'Neill B, Thiruganasambandamoorthy V, Parkash R, et al. Standardized approaches to the investigation of syncope: Canadian Cardiovascular Society position paper. Can J Cardiol. 2011;27:246-53.

18. Kapoor WN, Karpf M, Wieand S, Peterson JR, Levey GS. A prospective evaluation and follow-up of patients with syncope. $\mathrm{N}$ Engl J Med. 1983;309:197-204.

19. Cheshire WP. Syncope. Continuum (Minneap Minn). Selected Topics in Outpatient Neurology. 2017;23:335-58. 